\title{
Dietary supplementation with Withania somnifera root powder ameliorates experimentally induced Infectious Bursal Disease in chicken
}

\author{
Bhaskar Ganguly ${ }^{1,2}$ (D) Meena Mrigesh ${ }^{3} \cdot$ Premlata Chauhan $^{1} \cdot$ Sunil Kumar Rastogi ${ }^{1}$ \\ Received: 13 January 2019 / Accepted: 9 October 2019 / Published online: 9 November 2019 \\ (C) Springer Nature B.V. 2019
}

\begin{abstract}
Infectious Bursal Disease (IBD) is an economically important, immunosuppressive viral disease of chicken. Withania somnifera, a well-known Indian medicinal plant and functional food, finds extensive ethnomedicinal and ethnoveterinary use in the subcontinent. Root extracts of Withania somnifera have been shown to inhibit IBD virus (IBDV) in vitro. The effect of dietary supplementation with whole root powder of Withania somnifera was studied in chicken experimentally infected with IBDV. Dietary supplementation with the root powder improved erythrocytic indices, biochemical parameters, bursal weight index, and lymphocyte stimulation indices, and reduced histopathological insult in the infected birds. Viral load decreased to less than onefourth in the birds receiving dietary supplementation with Withania somnifera root powder. It could be concluded that continued supplementation of IBDV-infected chicken with Withania somnifera root powder alleviated virus-induced stress and histological and immunological alterations and reduced viral persistence in the host.
\end{abstract}

Keywords Infectious Bursal Disease $\cdot$ Indian ginseng $\cdot$ Withania somnifera $\cdot$ Stress $\cdot$ Physiology $\cdot$ Immunity

\section{Introduction}

IBD is an acute, highly contagious, and immunosuppressive viral disease of young chicken. The disease is characterized by the destruction of dividing lymphoid cells in the bursa of Fabricius leading to immunosuppression in addition to losses due to impaired growth, death, and excessive condemnation of carcasses due to skeletal muscle hemorrhages (Eterradossi and Saif 2013). Like most viral diseases of poultry, treatment is not available and vaccination is the principal method of prevention (Muller et al. 2012). However, vaccination against IBDV is

Bhaskar Ganguly

vetbhaskar@gmail.com

1 Animal Biotechnology Center, Department of Veterinary Physiology and Biochemistry, Govind Ballabh Pant University of Agriculture and Technology, Pantnagar, India

2 Present Address: Clinical Research Department, Research and Development Division, Ayurvet Limited, Katha, Baddi 173205, India

3 Department of Veterinary Anatomy, College of Veterinary and Animal Sciences, Govind Ballabh Pant University of Agriculture and Technology, Pantnagar 263145, India problematic. Inactivated and subunit vaccines result in different levels of maternally derived antibodies (MDA) in the offspring and compartmentalize the flock into individuals with low and high susceptibility to virulent IBDV. Live attenuated vaccines entail the risk of being neutralized by the MDA. Moreover, due to poor efficiency, such vaccines need to be applied at least twice, which is a labor-intensive and costly exercise. The use of more virulent live vaccines to overcome the latter problem itself causes immunosuppression and bursal damage (Arnold et al. 2012).

The use of various immuno-potentiating agents is therefore recommended both for reducing the effects of IBD and for improving host responses to vaccination with live viruses. Withania somnifera (Linn.) Dunal, commonly known as Indian ginseng or Indian wintercherry, is an important herb and functional food in many indigenous systems of medicine including Ayurveda, Unani, Siddha, Sowa Rigpa, and Chinese medicine. Popularly identified by its Sanskrit name Ashwagandha, it is regarded as the Queen of all Ayurvedic herbs, with its first use recorded approximately 5000 years ago (Tiwari et al. 2014). Roots of the plant are classified as an adaptogen and reputed to promote health and longevity (Weiner and Weiner 1994). The roots of the plant form a principal component of almost every herbal anti-stressor supplement for animals and humans in the subcontinent. Dietary 
supplementation with the Withania somnifera root powder (WSR) improves physiological functions in chicken suffering from heat stress, heavy metal toxicity, etc. Supplementation of broiler feed with WSR results in significant improvements in packed cell volume, hemoglobin percent, and hemagglutination inhibition titer against Newcastle disease virus, and better cellular response to dinitrochlorobenzene. WSR supplementation at $1 \%$ in feed significantly improves body weights, feed efficiency, and immune status of birds (Akotkar et al. 2007). Dietary supplementation of broilers with WSR at $0.1 \%$ in feed reverses cadmium-induced oxidative damage and restored hepatorenal functions and body weight; erythrocyte SOD and CAT activities are reduced, and GSH is increased besides reducing TBARS of the liver and renal tissues (Bharavi et al. 2010). Similarly, the supplementation of crude extract of Withania somnifera in broilers causes an effective and dosedependent reversal of enrofloxacin-induced immunosuppression (Arivuchelvan et al. 2013). Previous studies have confirmed that mono- and polyherbal preparations containing WSR improve host responses toward IBD and Newcastle disease vaccination. WSR also ameliorates viral chicken anemia in poultry (Tiwari et al. 2014). We were the first to show the in vitro inhibition of IBDV by an extract of Withania somnifera roots (Ganguly et al. 2010). Of late, a part of this inhibition has been attributed to its ability to induce nitric oxide production in the virus-infected host cells (Ganguly et al. 2018). Here, we report the results of an exploratory study on the ameliorative effects of dietary supplementation with WSR on IBDV-infected chicken.

\section{Materials and methods}

\section{Withania somnifera roots}

For a detailed description of the characterization of the roots of Withania somnifera used in the present study, the reader is referred to Ganguly et al. (2017). The dried roots were ground to a fine powder (mesh size 20) in a mechanical grinder for use.

\section{IBD virus and hyperimmune serum}

Pre-confirmed, chicken embryo fibroblast (CEF)-adapted IBD virus of strain UA-Bz 1 , passage 6 , available in the Animal Biotechnology Center, Pantnagar, was used in this study. The virus was exalted by three repeated passages in susceptible birds prior to inoculation in the test group of birds. The hyperimmune serum was raised against a live, invasive vaccine of IBDV (IV 95; Indovax Pvt. Ltd., India) by the standard method (Kumar et al. 2008).

\section{Experimental design}

All procedures involving animal experimentation, including those in the "IBD virus and hyperimmune serum" section, were in accordance with Breeding of and Experiments on Animals (Control and Supervision) Amendment Rules, Government of India, 2001, and were approved by the Institutional Animal Ethics Committee (Approval No. IAEC/VPB/CVASc/167).

In vivo experimentation was undertaken in 5-day-old White Leghorns $(N=48)$ of either sex, reared individually under cage system. The test comprised of a two-factorial de$\operatorname{sign}(n=12)$ as under:

\begin{tabular}{|c|c|c|}
\hline \multirow[t]{2}{*}{ WS } & \multicolumn{2}{|l|}{ Virus } \\
\hline & - & + \\
\hline- & Control (C) & Virus infected (V) \\
\hline+ & $\begin{array}{l}\text { Withania somnifera } \\
\text { supplemented (WS) }\end{array}$ & $\begin{array}{l}\text { Withania somnifera supplemented and } \\
\text { virus infected (WV) }\end{array}$ \\
\hline
\end{tabular}

Five-day-old White Leghorn chicks were obtained from Instructional Poultry Farm, Pantnagar, and allowed to acclimatize to the experimental conditions. At 1 week of age, the birds were randomly assigned to one of the four groups, and birds of groups WS and WV started to receive WSR at $1 \%$ of the feed. At 4 weeks of age, birds of groups $\mathrm{V}$ and $\mathrm{WV}$ received a total of $0.25 \mathrm{~mL}$ viral suspension, containing about $1.7 \times 10^{4} \mathrm{TCID}_{50}$ of IBDV, through oral, intraocular, intranasal, and intra-cloacal routes; the birds of groups $\mathrm{C}$ and WS were mock infected with buffered saline. Four birds from each group were randomly selected and exsanguinated on days 3 , 5 , and 7 post-infection.

\section{Absolute growth rate, feed conversion ratio, and hematology}

Live body weight was recorded on days 3,5 , and 7 postinfection, using a pan balance, and used to calculate the absolute growth rate. Feed consumption by the birds was recorded on a weekly basis and used to calculate the group-wise feed conversion ratio.

Blood was collected in heparinized vials by cardiac puncture and all tests were performed on pooled group samples in triplicate. Hemoglobin $(\mathrm{Hb})$ and packed cell volume (PCV) were determined using Sahli's hemoglobinometer and microhematocrit methods, respectively. Total erythrocyte count (TEC) and total leucocyte count (TLC) were determined by the method of Natt and Herrick (1952). Erythrocytic indices, i.e., mean corpuscular hemoglobin $(\mathrm{MCH})$, mean corpuscular volume (MCV), and mean corpuscular hemoglobin concentration $(\mathrm{MCHC})$, were deduced from the $\mathrm{Hb}, \mathrm{PCV}$, and TEC values. Differential leucocyte counts were made on 
monolayer blood films fixed and stained with GiemsaWright's stain.

Total protein, albumin, glucose, cholesterol, creatinine, aspartate aminotransferase (AST), and alanine aminotransferase (ALT) concentrations in plasma were determined by using commercial kits (Transasia Biomedicals Ltd., India). Plasma globulin was precipitated using ammonium sulfate and estimated using the biuret method (Chauhan 1998).

\section{Post-mortem findings and histopathology}

After exsanguination, the carcass weight was recorded and necropsy was performed. Bursal and splenic indices were determined and expressed as a percent of body weight. The bursa, spleen, and kidney were fixed in $10 \%$ formaldehyde (in PBS, $\mathrm{pH}$ 7.2) and stained with $\mathrm{H} \& \mathrm{E}$ as per standard method (Fischer et al. 2008) and sections were observed for histopathology. Bursal lesion scores were determined as per the method of Muskett et al. (1979). The mean of bursal lesion scores, assessed independently by three different persons, was used for comparison between the groups.

\section{Virological assays}

RT-PCR Total RNA was extracted from bursal tissue and reverse transcribed. A 249-bp fragment of $v p 2$ gene of IBDV was amplified using the primers pVP2_fw (5'-TGTT CTCAGCTAATATCGATGCCA-3') and pVP2_rv (5'-TCTC CAGTTTGATGGATGTGAT-3'). The primers used for amplifying a 194-bp fragment of the chicken $\beta$-actin gene were pAct_fw (5'-CTGACTGACCGCGTTACTCC-3') and pAct_rv (5'-ACCAACCATCACACCCTGAT-3'). The amplification products were visualized by electrophoresis in ethidium bromide-stained $1 \%$ agarose gel, and relative semi-quantification was performed using integrated densitometric values (IDV) (Chandra et al. 2014) determined by ImageJ v.1.48 (Abramoff et al. 2004).

Indirect immunofluorescence test Fixed tissue sections were dewaxed, rehydrated with PBS (pH 7.2), and left covered with $200 \mu \mathrm{L}$ of hyperimmune serum (1:500 in blocking buffer) for $1 \mathrm{~h}$ at $37^{\circ} \mathrm{C}$. The sections were washed thrice with PBS, $5 \mathrm{~min}$ per wash, and $200 \mu \mathrm{L}$ of secondary anti-chicken IgY-FITC conjugate was added. The sections were rewashed thrice with PBS, 5 min per wash, mounted on a glass slide, and covered with a coverslip. The sections were visualized under a fluorescent microscope at $\times 400$ magnification.

\section{Immunological assays}

Lymphocyte stimulation test Blood was diluted with four volumes of calcium- and magnesium-free PBS and $20 \mu \mathrm{L}$ of the resulting suspension was added to $200 \mu \mathrm{L}$ of growth medium containing $5 \mu \mathrm{g} / \mathrm{mL}$ of either concanavalin A (ConA) or lipopolysaccharide (LPS) to induce blastogenesis and allow lymphocyte proliferation. After $48 \mathrm{~h}$, MTT assay was performed and the stimulation index (SI) was calculated as per the following formula.

$\mathrm{SI}=\frac{\mathrm{Abs}_{\text {stimulated }}}{\mathrm{Abs}_{\text {unstimulated }}} ;$

where $\mathrm{Abs}_{\text {stimulated }}=$ mean corrected absorbance of wells stimulated with mitogen and $\mathrm{Abs}_{\text {unstimulated }}=$ mean corrected absorbance of unstimulated wells.

Thereafter, percent change (potentiation or suppression) in lymphocyte blastogenesis in response to a mitogen was calculated as follows:

Percent change $=\left(\frac{\mathrm{SI}_{\text {test }}-\mathrm{SI}_{\text {control }}}{\mathrm{SI}_{\text {control }}}\right) \times 100 \%$;

where $\mathrm{SI}_{\text {test }}=$ stimulation index of the test group, i.e., group V, $\mathrm{WS}$, or $\mathrm{WV}$, and $\mathrm{SI}_{\text {control }}=$ stimulation index of the control group, i.e., group $\mathrm{C}$.

Phagocytosis assay Blood, obtained on day 7 post-infection with the virus, was diluted with an equal volume of PBS $(\mathrm{pH}$ 7.2) and the resulting suspension was layered over half volume of HiSep-1119 in a centrifuge tube. The tube was spun in a hanging bucket centrifuge at $400 \mathrm{~g}$ for $20 \mathrm{~min}$ and the opaque, white layer containing the monocytes was collected.

$0.2 \mathrm{~mL}$ of this suspension was incubated at $37^{\circ} \mathrm{C} \times 30 \mathrm{~min}$ with $0.2 \mathrm{~mL}$ of $0.3 \%$ nitroblue tetrazolium (NBT; in PBS, $\mathrm{pH}$ 7.2) and $0.1 \mathrm{~mL}$ of activated plasma. The suspension was spun at $500 \mathrm{~g} \times 5 \mathrm{~min}$ and the supernatant was discarded. The cell pellet was washed and re-suspended in PBS (pH 7.2). A drop of the suspension was taken on a glass slide, a coverslip placed over it and observed under a microscope. NBT-positive cells were differentiated on the basis of the presence of blue-black granules of the dye and expressed as a percentage of the total number of cells counted.

\section{Statistical analyses}

All statistical operations were performed in MS Excel 2007. Statistical comparisons between different groups were made by analysis of variance (ANOVA) followed by post hoc Tukey test performed with Daniel's XL Toolbox add-in v.6.53. Unless stated otherwise, all statistical inferences were drawn at $P<0.01$.

\section{Results and discussion}

Infection with the virus deterred growth and reduced body weight (Table 1); this negative effect on growth rate and body 


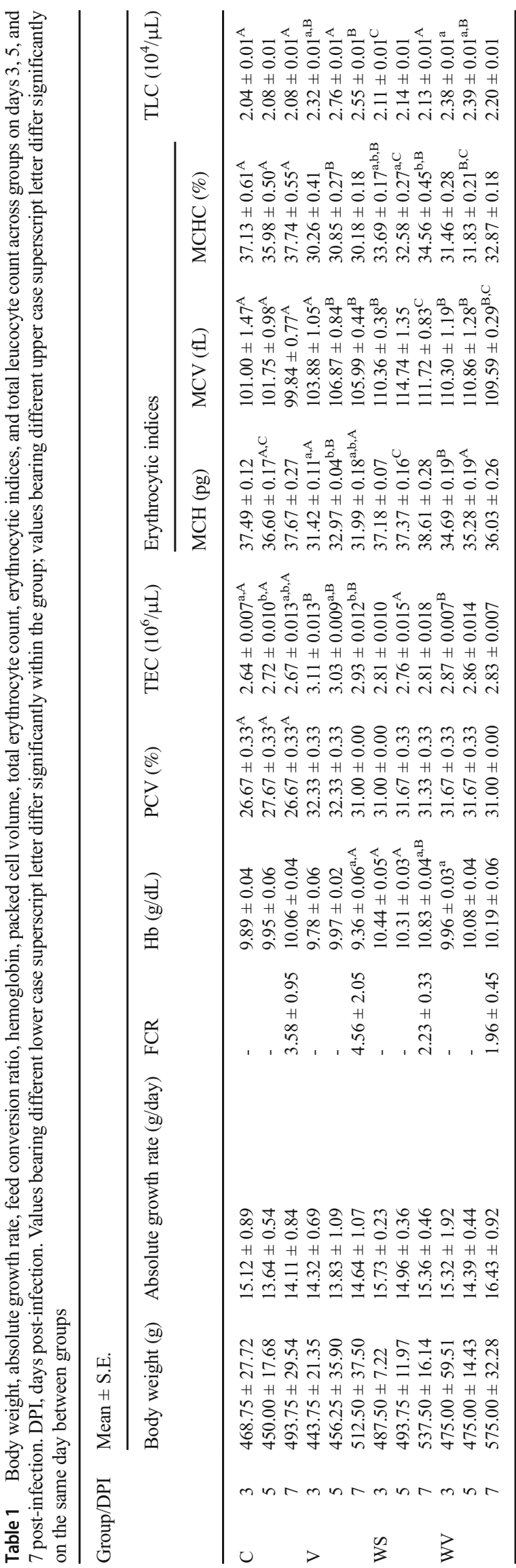

weight was ameliorated in birds receiving WSR in the feed. However, these differences in body weight and absolute growth rate between groups were non-significant $(P>0.01)$. Infection with the virus appeared to deteriorate the feed conversion ratio, the effect being lesser in the WV group. Birds receiving dietary supplementation with the root powder, irrespective of viral infection, had better feed conversion; however, all differences in feed conversion ratio were nonsignificant.

Akotkar and co-workers (Akotkar et al. 2007) compared the effect of supplementation of WSR in the feed of broilers at $0.50,0.75,1.00$, and $1.25 \%$, respectively, and found that supplementation at $1 \%$ of feed significantly improved body weight gain and feeding efficiency. WSR supplementation, at as little as $0.1 \%$ of feed, improves body weight gain in broilers experimentally subjected to cadmium toxicity (Bharavi et al. 2010). Significant improvements in body weight, feed conversion ratio, and performance index, subsequent to dietary supplementation with WSR at $1 \%$, have also been reported in broilers by Biswas et al. (2012).

Group $\mathrm{V}$ birds showed a reduction in $\mathrm{Hb}$ concentration, which worsened significantly $(P<0.01)$ on day 7 post-infection. Birds of group WV had improved Hb levels; however, within the group, the concentration was lowest on day 3 postinfection, which improved significantly by days 5 and 7 postinfection. Between groups, the WS group consistently showed the highest levels of $\mathrm{Hb}$. The results are summarized in Table 1. Akotkar et al. (2007) also reported significant increases in $\mathrm{Hb}$ content of all groups of broilers receiving WSR at varying concentrations in the diet.

PCV of the four groups is compared in Table 1. Between groups, the lowest $\mathrm{PCV}$ was found in the control group $\mathrm{C}$; the remaining three groups had comparably similar PCV. Previously, a strong decline up to 2 days post-infection followed by a semi-restorative increase up to 6 days post-infection in hematocrit was reported in Leghorns infected with IBDV at 4 weeks of age (Cheville 1967). Akotkar et al. (2007) have reported a significant increase in PCV of broilers receiving dietary supplementation of WSR; however, this was not observed in the present study.

Total erythrocyte count (Table 1) was significantly lower in the control group and significantly higher in the group V. Within the virus-infected group, highest TEC was present on the third day post-infection which declined gradually on days 5 and 7 post-infection. Based on the previous findings on $\mathrm{Hb}$ concentration and PCV, it appears that infection with IBDV causes the release of a large number of immature erythrocytes, resulting in elevated TEC and PCV accompanied by low $\mathrm{Hb}$ concentration. This effect of the virus was ameliorated in the birds of group $\mathrm{WV}$ as they showed improved $\mathrm{Hb}$ levels with a lesser number of erythrocytes and lower PCV.

$\mathrm{Hb}$ content, $\mathrm{PCV}$, and TEC were further used to determine the erythrocytic indices. The mean corpuscular hemoglobin 
concentration (Table 1) was the lowest in group $\mathrm{V}$ whereas group WV birds had significantly better $\mathrm{MCH}$ levels. $\mathrm{MCH}$ content in control and WS groups were similar to each other. Birds receiving WSR in feed, irrespective of virus infection, had higher MCV (Table 1). On day 3 post-infection, the MCV of groups WS and WV were significantly higher than the MCV of control and V groups. On day 5 post-infection, the WS group had the highest MCV whereas groups WV and V had significantly higher MCV than control. On day 7 postinfection, the birds of group WS had a non-significantly higher MCV than the birds of the WV group, which also had non-significantly higher MCV than the birds of group V. The differences in the MCV of groups WS and V were significant and the control group had the significantly lowest MCV of the four groups. A comparison of the MCV values on day 7 post-infection reflected significantly higher values in the two WSR-supplemented groups and significantly lowest values in the control group.

Birds of the control group had significantly highest $\mathrm{MCHC}$, followed by birds of WS, WV, and V groups (Table 1). The trend almost persisted throughout days 3, 5, and 7 post-infection. The presence of hypochromic, macrocytic RBCs in the virus-infected group further corroborates the hypothesis that IBDV infection causes the release of immature erythrocytes in the circulation. Erythrocytic indices of the WV group suggest that this effect of the virus was countered, at least in part, by the dietary supplementation of WSR.

Total leucocyte count (Table 1) was significantly elevated in the virus-infected birds; the leukocytosis was more pronounced, but immediate and transient, in birds that had been receiving WSR in the feed. The WS group showed an increase, though not always significant, in TLC over the control group. Contrary to this finding, severe panleukopenia has been reported by Cheville (1967) on the fourth day post-infection in Leghorns infected with IBDV at 4 weeks of age.

Birds of the WS group had consistently lowest heterophil percentage (Fig. 1), while the virus-infected group appeared to have continuing monocytosis. Also, on day 5 post-infection, birds of group $\mathrm{V}$ showed a marked increase in heterophil percentage, with an accompanying decrease in lymphocyte percentage. Cheville (1967) reported a pronounced lymphocytopenia on days 2 and 3 post-infection, which returned to normalcy by about ninth day post-infection, in Leghorns infected with IBDV at 4 weeks of age.

Absolute leukocyte counts (Fig. 1) showed an elevation in the heterophil and lymphocyte counts upon infection with the virus. Birds of group WV showed a strong response to the viral infection immediately as compared with birds of group $\mathrm{V}$, where the response was slower. Moreover, the group V birds showed elevated heterophil to lymphocyte ratios, indicating a state of stress. Infection with the virus also appears to cause monocytosis, which was again lesser in the birds receiving WSR in the feed. The WS group had higher monocyte counts and lower heterophil to lymphocyte (H:L) ratios as compared with control. H:L ratio is a major indicator of stress in poultry (Puvadolpirod and Thaxton 2000).

Plasma protein concentration (Table 2), irrespective of virus infection, was higher in birds supplemented with WSR. Fifth day post-infection, there was a prominent increase in the total plasma protein level of group WV whereas on the seventh day post-infection, virus-infected birds showed a significant reduction in plasma protein concentration. Cheville

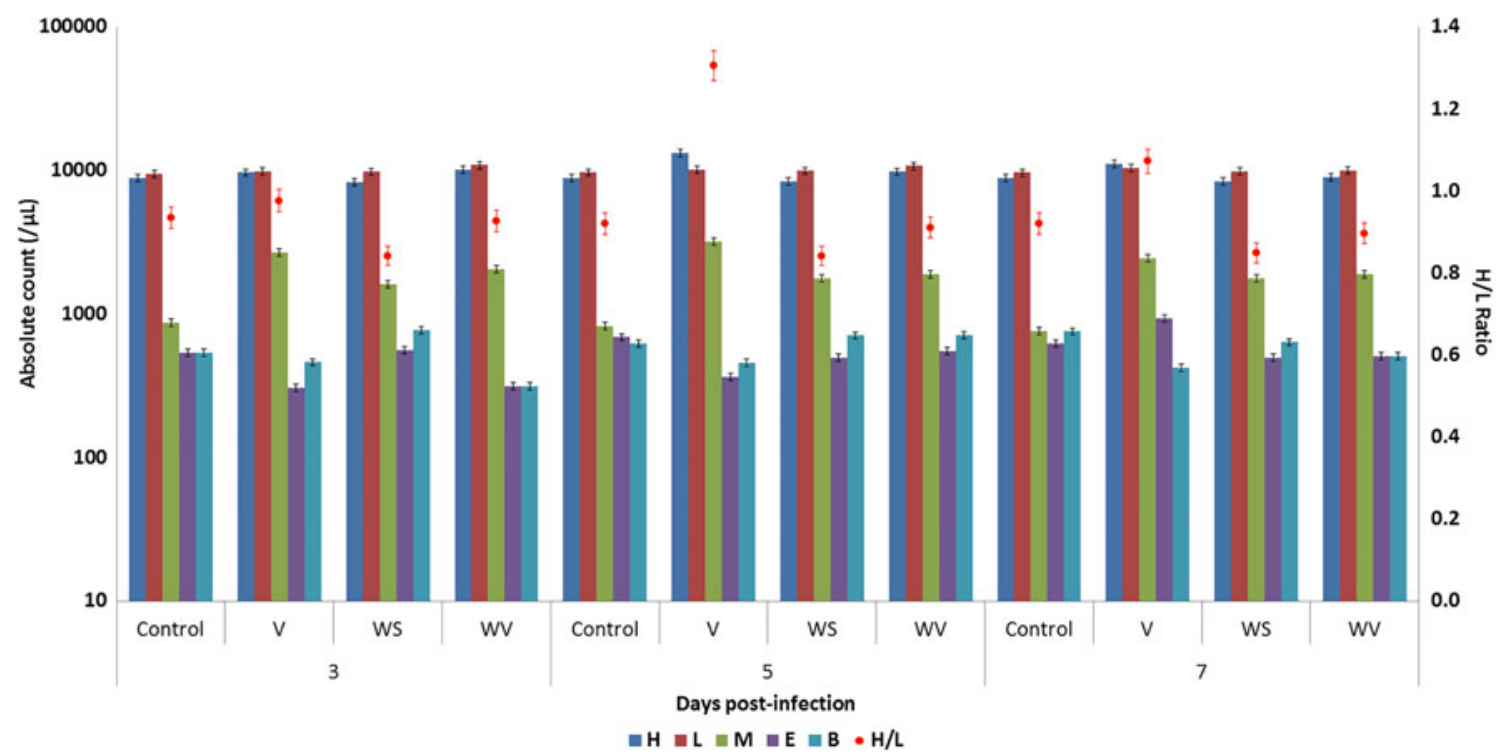

Fig. 1 Absolute leucocyte counts and heterophil to lymphocyte ratio in birds post-infection. H, heterophil; L, lymphocyte; M, monocyte; B, basophil; E, eosinophil 


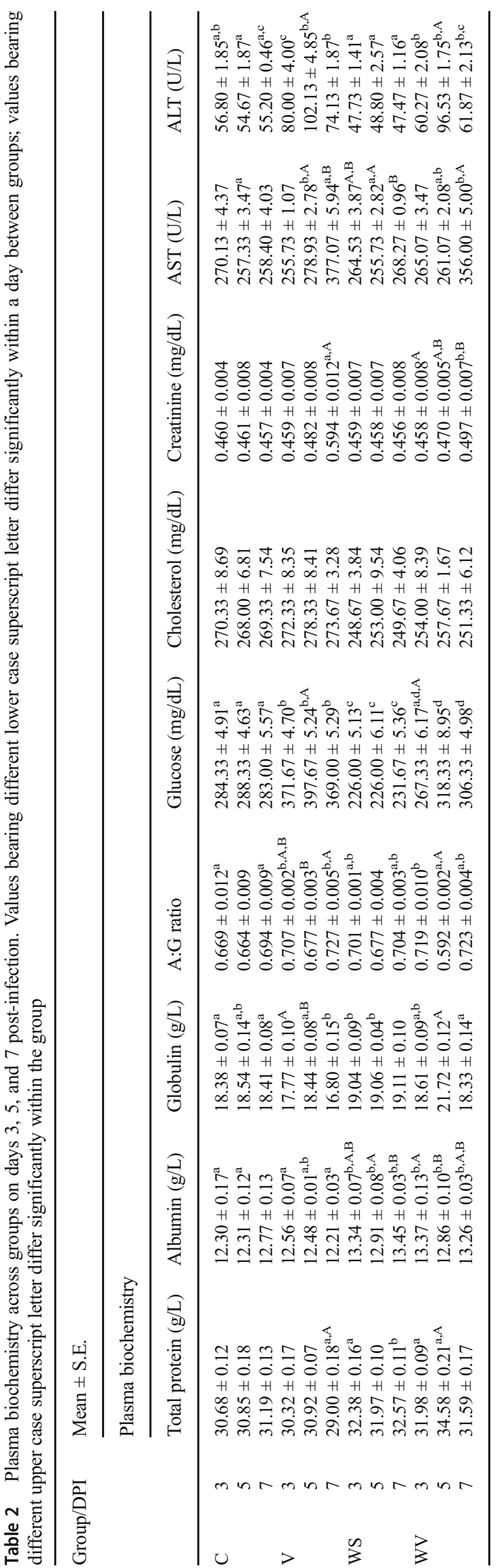

(1967) has reported an increase in serum protein concentrations on the sixth day post-infection in convalescent Leghorns that were infected with IBDV at 4 weeks of age. The significant increase in plasma proteins at 5 days post-infection may, thus, be indicative of rapid recovery from the viral insult in the WV group.

Plasma albumin concentration (Table 2) was significantly higher in both groups of WSR-supplemented birds. Within the group, the WV birds showed a significant decline in albumin concentration on day 5 post-infection. The hypoalbuminemia within group $\mathrm{V}$ was found non-significant. Transient hypoalbuminemia is known to occur during the severe exudative phase of IBD, as in many other diseases of the bowel. This is due to the loss of serum albumin during the inflammatory exudation into the bursa and subsequent excretion with fecal material (Cheville 1967).

Plasma globulin concentration (Table 2) was significantly higher in group WS on days 3 and 7 post-infection. On the fifth day post-infection, group WV birds had the highest globulin concentration. On the fifth day post-infection, birds of group $\mathrm{V}$ also showed a significant increase in plasma globulins. Overall, plasma globulin concentration was the lowest in the birds of group V. Cheville (1967) could not detect any alterations in globulins over a period of 2 weeks post-infection in Leghorns infected with IBDV at 4 weeks of age.

Albumin to globulin ratio (Table 2) was the lowest in birds of group WV on day 5 post-infection, due mainly to a concomitant, significant increase in globulin levels. Arivuchelvan et al. (2013) have reported that dietary supplementation with Withania somnifera crude extracts brought about a nearcomplete reversal of enrofloxacin-induced hypoproteinemia, hypoalbuminemia, and hypoglobulinemia in broilers. In chicken, the mean reference value of total serum proteins is $56 \mathrm{~g} / \mathrm{L}$ and the protein content of the plasma is about $1.5 \mathrm{~g} / \mathrm{L}$ higher than this value due to the added presence of fibrinogen (Kaneko et al. 1997; Lumeij 1997). Similarly, the mean reference values of albumin and globulin in the chicken are about $25 \mathrm{~g} / \mathrm{L}$ and $31 \mathrm{~g} / \mathrm{L}$, respectively (Kaneko et al. 1997). The finding in this study of an overall reduction in total protein and its subcomponents was consistent with previous works mentioned above. However, the A:G ratio appears to have remained conserved.

Plasma glucose concentration (Table 2) showed a steep increase in the virus-infected groups; within the groups, the concentration was highest on fifth day post-infection. The magnitude of hyperglycemia was significantly lower in birds receiving WSR feed. Between groups, the glucose concentration was consistently found lowest in the birds of the WS group. The mean reference value of glucose in chicken is $167.8 \mathrm{mg} / \mathrm{d}$; the range of glucose concentration, uncertain in chicken, is known to vary a lot in aves (Kaneko et al. 1997). It is unclear if the values of plasma glucose obtained in the 
present study represent a state of hyperglycemia or euglycemia.

Plasma cholesterol concentration (Table 2) was numerically higher in the birds of groups not receiving WSR in the diet. Between groups, cholesterol concentration was apparently lowest in the birds of WS group; cholesterol levels of WV group were closely matched; the statistical differences between any two individual values were non-significant. The mean reference value of cholesterol in chicken is $183.8 \mathrm{mg} /$ dL (Kaneko et al. 1997). Thus, cholesterol level also appears elevated in the study groups. A to G ratio, glucose, and cholesterol levels are indices of stress in chicken (Puvadolpirod and Thaxton 2000). A to G ratio, plasma glucose, and cholesterol were consistently elevated in group $\mathrm{V}$; the increment was lower in the birds of the WV group.

Plasma creatinine (Table 2) elevated upon virus infection; the increase was non-significant on day 3 post-infection but grew significant by day 7 post-infection. Group WV birds showed a lesser increase in creatinine levels as compared with group V birds. Previously, Bharavi et al. (2010) have reported WSR supplementation at $0.1 \%$ of the feed to cause a partial reversal of cadmium-induced elevation of creatinine levels in broilers.

The results of the assays for aspartate aminotransferase (AST) and alanine aminotransferase (ALT) are shown in Table 2. The levels of both enzymes in plasma were elevated significantly subsequent to IBDV infection. However, the trends of increase for the two enzymes were different. AST showed a gradual but continuous increase throughout the study period, showing a maximum concentration on day 7 post-infection. ALT showed an immediate increase, reaching maximum concentration on day 5 post-infection, and then declined on day 7 post-infection. Again, the increase in both enzymes studied was lesser in birds of group WV as compared with birds of group V. Bharavi et al. (2010) have reported WSR supplementation at $0.1 \%$ of the feed to cause a partial reversal of cadmium-induced elevation of ALT levels in broilers. The increased levels of plasma creatinine, AST, and ALT may be due to elusive damage to muscular and hepatic tissues by the virus because necropsy did not reveal any gross lesions; IBDV is known to cause lesions in these tissues (Eterradossi and Saif 2013).

Bursal indices are compared in Table 3 . On day 3 postinfection, the birds of groups WV and $\mathrm{V}$ showed a nonsignificant bursal enlargement while on days 5 and 7 postinfection, birds of group $\mathrm{V}$ showed atrophy. Within group V, the change in the bursal index between days 3 and 7 postinfection was highly significant. The overall differences were also highly significant. Cheville (1967) has reported a very strong increase in absolute bursal weights, peaking on the fourth day post-infection, followed by an overwhelming decrease continuing until about 8 days post-infection in Leghorns infected with IBDV at 4 weeks of age. However, the study does not state the changes in the body weight of the birds during this period. Group WS birds apparently had the smallest spleens of all four groups; however, all differences between splenic indices (Table 3) were found to be non-significant. Previously, Cheville (1967) has reported moderate to severe splenomegaly in IBDV-infected Leghorns.

Upon gross examination, bursae collected from birds on day 3 post-infection appeared congested with numerous petechiae. The amount of bursal colloid, copiously present in the bursae of group WS, was scarce in bursae of groups V and WV. Macroscopically, bursae of the WV group appeared pale and discolored yet healthier than those of group V. By the seventh day post-infection, bursae of group $\mathrm{V}$ were severely
Table 3 Organ weight indices, bursal lesion score, and phagocytic indices across groups on days 3,5 , and 7 post-infection. Phagocytic index and relative viral load were assessed only on day 7 post-infection. Values bearing different upper case superscript letter differ significantly within the group

\begin{tabular}{|c|c|c|c|c|c|}
\hline \multirow{3}{*}{\multicolumn{2}{|c|}{ Group/DPI }} & \multicolumn{4}{|l|}{ Mean \pm S.E. } \\
\hline & & \multicolumn{2}{|c|}{ Organ weight index } & \multirow[t]{2}{*}{ Bursal lesion score } & \multirow{2}{*}{$\begin{array}{l}\text { Phagocytic index } \\
\text { (\% NBT-positive cells) }\end{array}$} \\
\hline & & Bursa & Spleen & & \\
\hline \multirow[t]{3}{*}{$\mathrm{C}$} & 3 & $1.35 \pm 0.03$ & $0.81 \pm 0.06$ & 0 & - \\
\hline & 5 & $1.33 \pm 0.05$ & $0.81 \pm 0.04$ & 0 & - \\
\hline & 7 & $1.32 \pm 0.05$ & $0.79 \pm 0.11$ & 0 & $26.667 \pm 2.333$ \\
\hline \multirow[t]{3}{*}{ V } & 3 & $1.42 \pm 0.05^{\mathrm{A}}$ & $0.87 \pm 0.05$ & $1.00 \pm 0.00$ & - \\
\hline & 5 & $1.19 \pm 0.10^{\mathrm{A}, \mathrm{B}}$ & $0.93 \pm 0.05$ & $1.67 \pm 0.33$ & - \\
\hline & 7 & $0.99 \pm 0.05^{\mathrm{B}}$ & $0.96 \pm 0.04$ & $3.67 \pm 0.33$ & $21.667 \pm 2.333$ \\
\hline \multirow[t]{3}{*}{ WS } & 3 & $1.34 \pm 0.03$ & $0.74 \pm 0.02$ & 0 & - \\
\hline & 5 & $1.35 \pm 0.04$ & $0.75 \pm 0.04$ & 0 & - \\
\hline & 7 & $1.32 \pm 0.03$ & $0.69 \pm 0.04$ & 0 & $32.000 \pm 2.646$ \\
\hline \multirow[t]{3}{*}{ WV } & 3 & $1.53 \pm 0.21$ & $0.93 \pm 0.22$ & $1.33 \pm 0.33$ & - \\
\hline & 5 & $1.42 \pm 0.04$ & $0.85 \pm 0.06$ & $1.33 \pm 0.33$ & - \\
\hline & 7 & $1.32 \pm 0.06$ & $0.71 \pm 0.06$ & $2.33 \pm 0.33$ & $26.000 \pm 2.309$ \\
\hline
\end{tabular}


atrophic, whereas those from the WV group were normal in size. Histopathology (Fig. 2) and bursal lesion scoring (Table 3) corroborated these findings.

Figure 2 a shows the normal structure of bursa collected from a bird of the control group on day 3 post-infection. The bursal follicles consisted of medullary and cortical areas, separated by a single-celled layer of endodermal epithelium, which was continuous with the epithelium of bursal lumen. The medullary areas of the follicle contained small lymphocytes whereas the cortical areas contained large lymphocytes and cells of the plasmacyte lineage, which differentiate from the endodermal epithelium. This architecture remained conserved in the control group on days 5 and 7 post-infection and in the WS group on days 3,5 , and 7 post-infection.
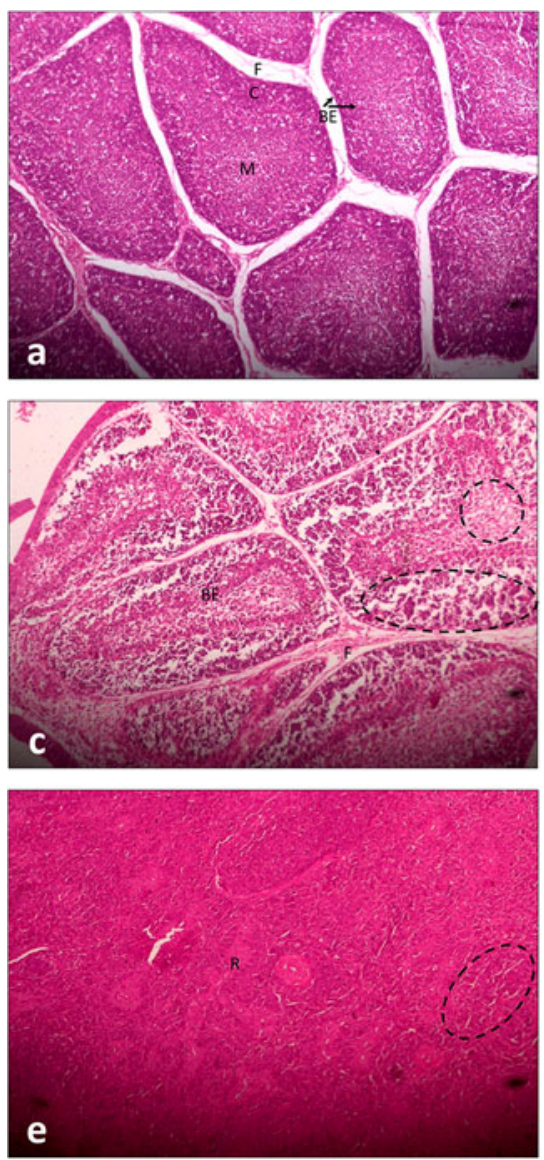

Fig. 2 Histopathological sections of bursae and spleen (H\&E staining, $\times$ 100). a Bursal section from control, third day post-infection; discreet bursal follicles with scanty inter-follicular fibrous tissue (F) and bound by a single-celled layer of bursal epithelium (BE) can be seen. The bursal epithelium also separates the cortex (C), containing large lymphocytes, from the medulla (M), which contains small lymphocytes. A similar structure was evident on days 5 and 7 post-infection; the histological appearances of sections obtained from bursae of group WS were indistinct and unremarkable. b Bursal section from group $\mathrm{V}$, seventh day postinfection; large amounts of fibrous connective tissue (F), severe lymphoid depletion (black dashed circles), reticular hyperplasia (R) and fatty degeneration (yellow dashed circles), and marked thickening of the
On the third day post-infection, there was an appreciable increase in the thickness of the endodermal epithelium accompanied by necrosis of lymphoid cells and lymphoid depletion in both medullary and cortical areas. Pale, large reticular cells had started to appear and a moderate degree of inter-follicular fibroplasia was also seen. On the fifth day post-infection, very severe fibroplasia that invaded the intra-follicular space was present in the bursa. In many areas, the bursal medulla became nearly depleted of small lymphocytes. The number of reticular cells was found to have increased as a response to clearing the dead lymphocytes, and a large number of heterophils also started appearing. By the seventh day post-infection (Fig. 2b), the state of intense
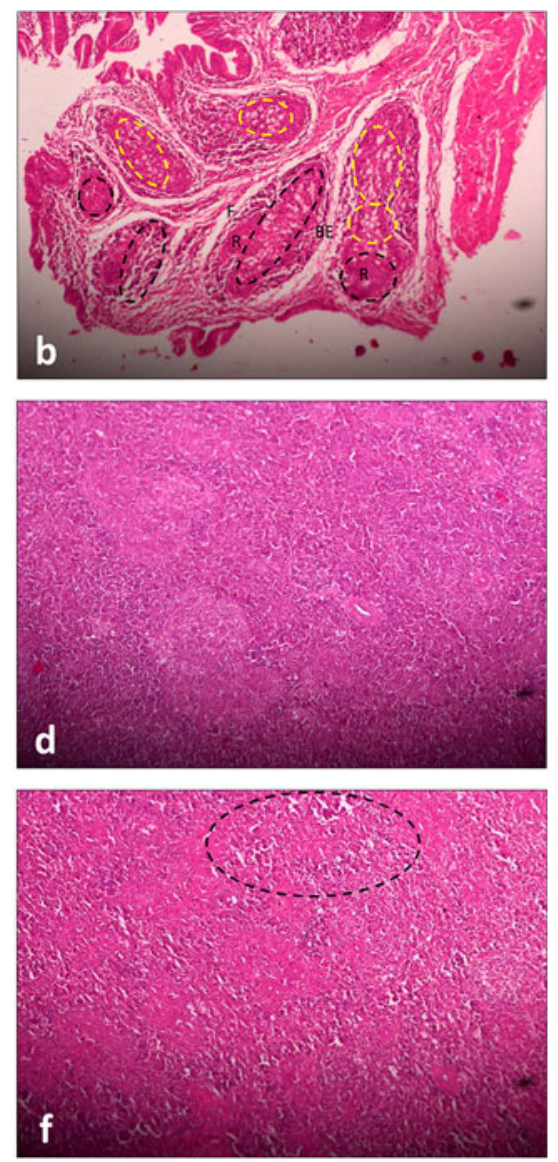

endodermal epithelium (BE) are evident. c Bursal section from group $\mathrm{WV}$, seventh day post-infection; fibrosis $(\mathrm{F})$, thickening of the bursal epithelium (BE), and lymphoid depletion (dashed circles) were less severe than those from group V. d Section of spleen from control, seventh day post-infection; the histological appearances of sections obtained from the spleen of group WS were indistinct. e Section of spleen from group V, seventh day post-infection; intense reticular hyperplasia (R) accompanying moderate lymphoid depletion (dashed circle) is evident. f Section of spleen from group WV, seventh day post-infection; moderate lymphoid depletion (dashed circle) can be seen throughout the section but reticular hyperplasia is absent 
fibroplasia had subsided leaving the inter-follicular spaces full of connective tissue. The follicles were severely depleted of lymphocytes; the medulla was almost completely obliterated by reticular hyperplasia and fatty degeneration, and the endodermal epithelium was also markedly thickened. However, the replacement of the endodermal bursal epithelium with columnar epithelium bearing mucin-producing, goblet-like cells, as described previously by Cheville (1967), was not seen. A good number of heterophils persisted, especially so in the cortical areas of the follicles. The virus-induced lymphonecrosis was milder with a more rapid reticular response in the bursae of birds of the WV group. On the seventh day post-infection (Fig. 2c), follicles of the bursa from the WV group were certainly depleted of lymphocytes, more so in the medullary areas, but the follicular morphology was still preserved with very limited fibrosis.

The histopathological alterations in the renal tissue of virus-infected birds were restricted to a slightly dilated appearance of tubules; no other changes were appreciable and sections from the remaining groups were identical otherwise. The histological sections of the spleen collected the seventh day post-infection from birds of different groups are shown in Fig. 2d-f. In normal spleen (Fig. 2d), lymphocytes surround the terminal arterioles forming the periarteriolar lymphoid sheath.
These arterioles then branch, becoming sheathed with phagocytic reticular cells and finally dilate to form sinuses of the red pulp. Germinal follicles, which consist of blast cells, lymphoblasts, and large lymphocytes, are structurally associated with splenic arterioles. The spleen from the virus-infected group (Fig. 2e) showed moderate lymphonecrosis with intense reticular hyperplasia. Similar findings have been reported previously by Cheville (1967). Although lymphoid depletion was present, reticular hyperplasia was limited in the spleen of birds of the WV group (Fig. 2f).

The mean bursal lesion scores ascertained independently by three different observers are shown in Table 3. By the seventh day post-infection, a large difference in the bursal lesion score was seen between groups V and WV. The overall differences between groups were highly significant.

Figure 3 shows the results of the determination of relative viral load in the bursae collected from the different groups on the seventh day post-infection on the basis of integrated densitometric values. The differences between control and WS groups were non-significant; all other differences were highly significant.

Figure 4 shows the immunofluorescence in sections of bursae collected from groups $\mathrm{V}$ and WV on day 7 post-infection. Immunofluorescence is a standard test for
Fig. 3 Relative semiquantification of viral load in bursae on the seventh day postinfection; IDV of RT-PCR product of 249-bp fragment of $V P 2$ RNA was used for the relative semi-quantification of viral load; IDV of RT-PCR product of $\beta$ actin transcript served as control

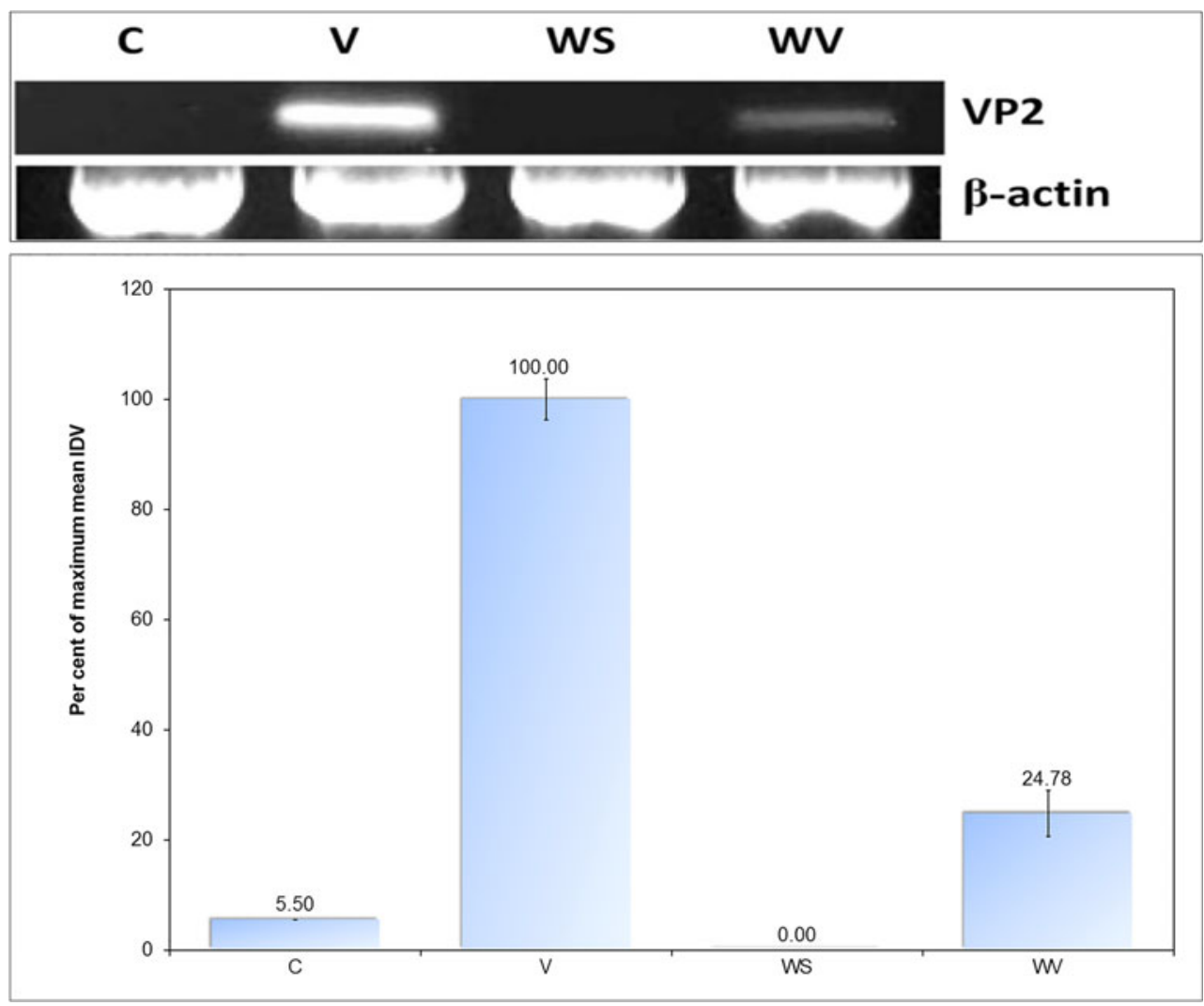



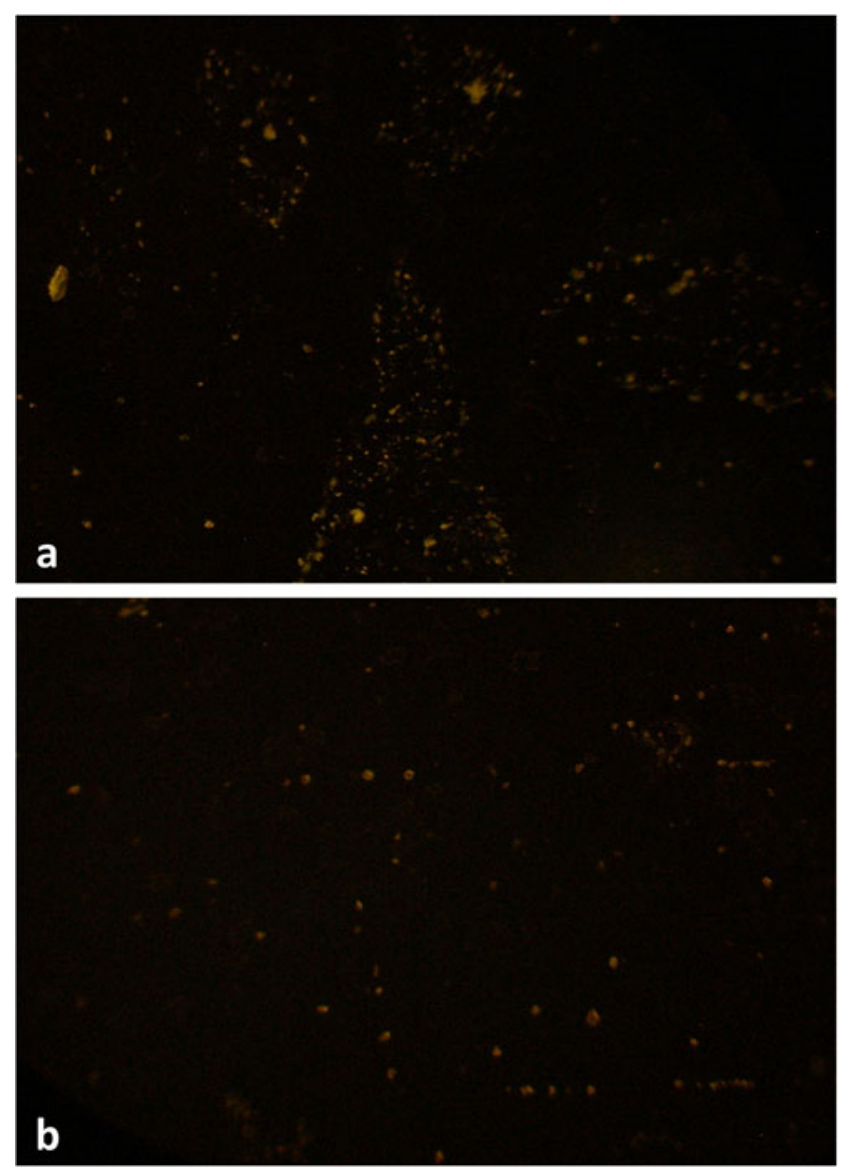

Fig. 4 Immunofluorescent staining of sections of bursae collected on day 7 post-infection $(\times 100)$. a Bursal section from group V shows higher fluorescence, concentrated within follicles. b Bursal section from group WV shows lesser and diffuse fluorescence

demonstrating the presence of the virus in host tissue (Eterradossi and Saif 2013). The bursal section from group $\mathrm{V}$ shows higher viral antigen concentrated mainly within the follicles; the follicular silhouette was apparent in the fluorescence pattern. The WV group showed lesser viral load that was diffusely present throughout the bursal section; however, few small, focal concentrations of viral antigen were still discernible.

Thus, birds of the WV group had better bursal histology and lower viral loads as compared with their unsupplemented counterparts. This may be due to the stronger immune response mounted by the birds of group WV and rapid neutralization of virus, as suggested by higher globulin concentration and a higher number of circulating lymphocytes.

The differences in mitogenic response to ConA and LPS (Fig. 5) were highly significant between groups but the differences between days were found non-significant. Infection with the virus appeared to reduce the responsiveness of both $\mathrm{B}$ and $\mathrm{T}$ lymphocytes to mitogens; this is in agreement with the previous findings of Confer and MacWilliams (1982). It has been suggested that suppression in $\mathrm{T}$ cell mitogenesis may not be due to direct viral effects on $\mathrm{T}$ cells and the depression of mitogen responsiveness seen may be an indication of immature lymphocytes released into circulation (Confer and MacWilliams 1982). Again, birds of the WV group showed better mitogen responsiveness than birds of group V.

Phagocytic functions (Table 3) were also apparently better in the WV group than in group V; however, the differences were found non-significant. Previously, Akotkar et al. (2007) have reported improved HI titers to Newcastle disease vaccine and a stronger cell-mediated cutaneous response to dinitrochlorobenzene in broilers receiving WSR in the diet. Arivuchelvan et al. (2013) also reported crude Withania somnifera extract supplementation to improve diminished HI titers to Newcastle disease vaccine in enrofloxacin-treated broilers. A significant increase in CD69 expression and an absolute number of $\mathrm{CD} 8^{+} \mathrm{T}$ cells, along with an overall trend toward increased activation of $\mathrm{CD} 4^{+} \mathrm{T}$ cells, has been reported in human subjects consuming $3 \mathrm{~mL}$ of Withania somnifera root extract with organic cow milk twice daily for 5 days (Erlandsen et al. 2012).

In summary, IBDV-infected chicken receiving dietary WS supplementation (group WV) had significantly improved hemoglobin and mean corpuscular hemoglobin, higher mean corpuscular volume, transient leukocytosis, and faster heterophilia and lymphocytosis, as compared with unsupplemented virus-infected birds (group V). Birds of group WV also had significantly improved total protein, albumin, and globulin levels in plasma as compared with birds of group V. The birds of group V developed elevated plasma levels of glucose, creatinine, aspartate aminotransferase (AST), and alanine aminotransferase (ALT) post-infection. The level of these analytes was significantly better in group WV. Birds of group WV failed to show a significant improvement in bursal index, though gross and histological appearance of the bursae were qualitatively much better in this group than those from group V. Relative semiquantitation of the viral antigen in bursae revealed that group WV had less than one-fourth $(24.78 \%)$ of the viral load in group $\mathrm{V}$ by day 7 post-infection. In the indirect immunofluorescent test, bursae from group WV again showed a qualitative reduction in viral antigen as compared with the bursae from group V. Birds of group $\mathrm{V}$ showed significantly lowered responsiveness of B and $\mathrm{T}$ lymphocytes to mitogens. Mitogenic responsiveness of lymphocytes was significantly better in the birds of group WV than in the birds of group V. The birds of group WV also showed non-significant improvements in several other parameters such as total body weight, 

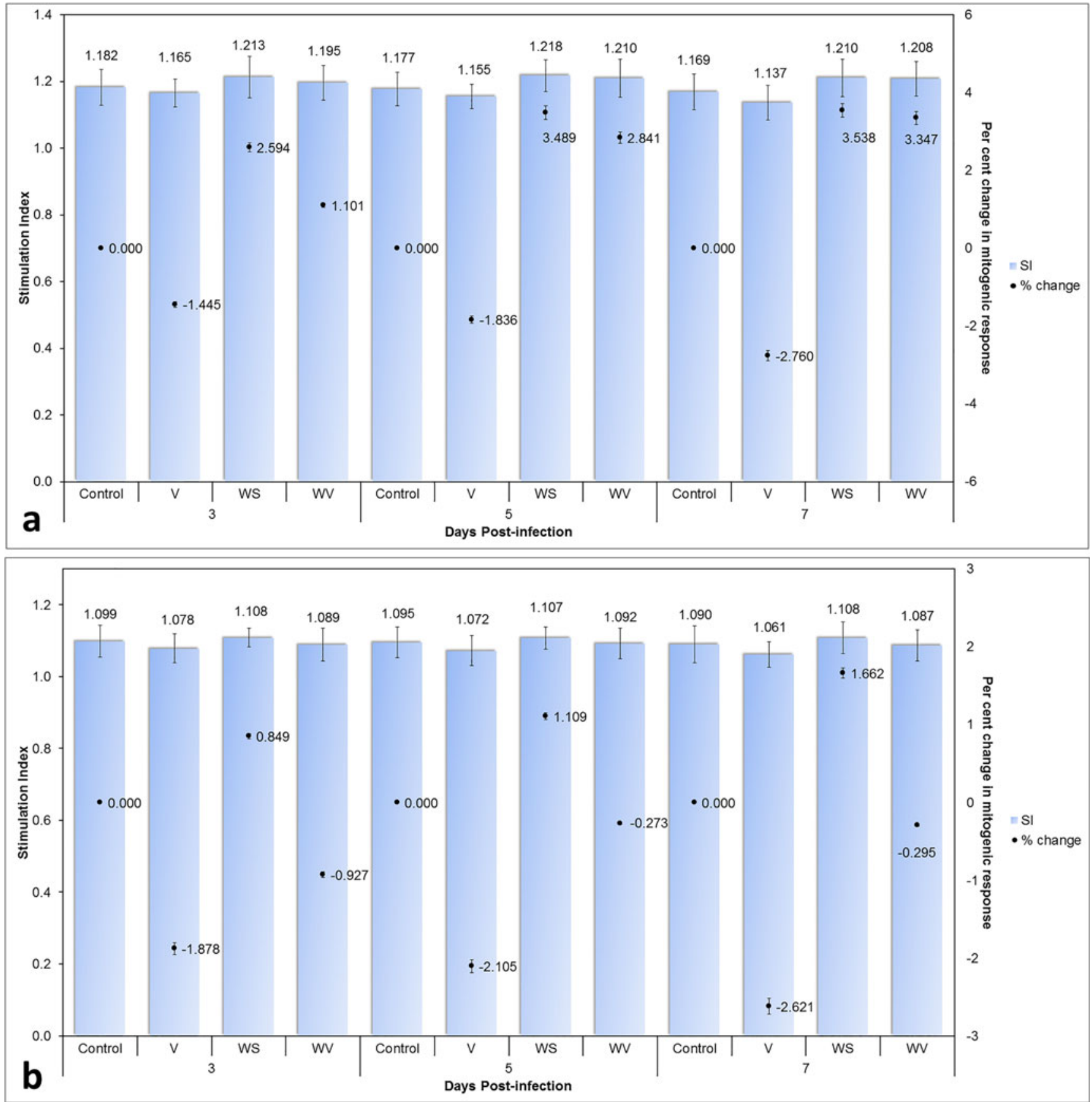

Fig. 5 The mitogenic response of lymphocytes to stimulation with a concanavalin A and b lipopolysaccharide; stimulation index (SI) and percent change in the mitogenic response have been shown for comparison

growth rate, feed conversion ratio, plasma cholesterol concentration, and macrophage function as compared with the birds of group V. The study concluded that continued dietary supplementation of layers with WS root powder at $1 \%$ of feed ameliorates IBDV-induced hematological, biochemical, and immunological alterations, resulting in lower viral persistence in the host, and also afforded protection against virus-induced stress and histopathological changes. Thus, dietary supplementation of chicken with WS root powder holds promise for protecting the chicken from Infectious Bursal Disease, reducing morbidity in infected birds as well as for combating immunosuppression in birds immunized with a hot vaccine.

Acknowledgments The authors thank Drs. S. Kumar, A. K. Das, A. K. Upadhyay, S. K. Shukla, S. Vatsya, and M. K. Saxena of G.B.P.U.A. \& T., Pantnagar, for facilitating different phases of the study. 
Funding information BG received financial support during this study in the form of a Senior Research Fellowship (Ref No. 20-06/2010(i)EU-IV) from the Council of Scientific and Industrial Research, India.

Compliance with ethical standards All institutional and national guidelines for the care and use of laboratory animals were followed.

Conflict of interest The authors declare that they have no conflict of interest.

\section{References}

Abramoff MD, Magelhaes PJ, Ram SJ. 2004. Image Processing with ImageJ. Biophotonics Int, 11:36-42.

Akotkar NS, Sarag AN, Rekhate DH, Dhok AP. 2007. Effect of supplementation of Ashwagandha (Withania somnifera) on performance of broilers. Indian J Poultry Sci, 42:92-94.

Arivuchelvan A, Murugesan S, Mekala P. 2013. Immunomodulatory effect of Withania somnifera in broilers treated with high doses of enrofloxacin. Indian J Drugs Dis, 2:276-279.

Arnold M, Durairaj V, Mundt E, Schulze K, Breunig KD, Behrens SE. 2012. Protective vaccination against infectious bursal disease virus with whole recombinant Kluyveromyces lactis yeast expressing the viral VP2 subunit. PloS One, 7:e42870.

Bharavi K, Reddy AG, Rao GS, Reddy AR, Rao SVR. 2010. Reversal of Cadmium-induced oxidative stress in chicken by herbal adaptogens Withania somnifera and Ocimum sanctum. Toxicol Int, 17:59-63.

Biswas P, Sharma RK, Biswas A. 2012. Effect of dietary supplementation of Ashwagandha (Withania somnifera) and ascorbic acid on production performance of broiler birds. Indian J Poultry Sci, 47:403-405.

Chandra S, Narang R, Sreenivas V, Bhatia J, Saluja D, Srivastava K. 2014. Association of Angiotensin II Type 1 Receptor (A1166C) Gene Polymorphism and Its Increased Expression in Essential Hypertension: A Case-Control Study. PLoS One, 9:e101502.

Chauhan RS. 1998. Measurement of humoral immune response In: Laboratory Manual of Immunopathology. G. B. Pant University of Agriculture and Technology, Pantnagar; 37.

Cheville NF. 1967. Studies on the pathogenesis of Gumboro disease in the bursa of Fabricius, spleen, and thymus of the chicken. American J Pathol, 51:527.

Confer AW, MacWilliams PS. 1982. Correlation of hematological changes and serum and monocyte inhibition with the early suppression of phytohemagglutinin stimulation of lymphocytes in experimental infectious bursal disease. Canadian J Comp Med, 46(2):169.

Erlandsen A, Mikolai J, Murison A, Mukherjee S, Zwickey H. 2012. In vivo immune modulating effects of Ashwagandha (Withania somnifera). BMC Complementary Alt Med, 12(Suppl 1):P44.
Eterradossi N, Saif YM. 2013. Infectious bursal disease. In: Swayne DE, Glisson JR, McDougald LR, Nolan LK, Suarez DL, Nair V. eds. Diseases of Poultry $13^{\text {th }}$ edn. Wiley Blackwell. pp. 219-246.

Fischer AH, Jacobson KA, Rose J, Zeller R. 2008. Hematoxylin and eosin staining of tissue and cell sections. Cold Spring Harbor Protocols, 2008(5):pdb-prot4986.

Ganguly B, Kumar S, Umapathi V, Ambwani T. 2010. Inhibition of Gumboro virus by Withania somnifera extract. In: $5^{\text {th }}$ Uttarakhand State Science and Technology Congress Compendium of Abstracts, Uttarakhand Council for Science and Technology, Dehradun, pp. 216.

Ganguly B, Kumar N, Ahmad AH, Rastogi SK. 2017. Influence of phytochemical composition on in vitro antioxidant and reducing activities of Indian ginseng [Withania somnifera (L.) Dunal] root extracts. J Ginseng Res, doi:/10.1016/j.jgr.2017.05.002

Ganguly B, Umapathi V, Rastogi SK. 2018. Nitric oxide induced by Indian ginseng root extract inhibits Infectious Bursal Disease virus in chicken embryo fibroblasts in vitro. J Ani Sci Technol, 60:2. https://doi.org/10.1186/s40781-017-0156-2

Kaneko JJ, Harvey JW, Bruss ML. 1997. Clinical Biochemistry of Domestic Animals. $5^{\text {th }}$ ed. Harcourt Brace and Company, Singapore. pp. 900-901.

Kumar A, Umapathi V, Singh R. 2008. Production of Antiserum. In: Techniques of Immunology and Serology. G. B. Pant University of Agriculture and Technology, Pantnagar; pp. 17-30.

Lumeij JT. 1997. Avian Clinical Biochemistry. In: Kaneko JJ, Harvey JW, Bruss ML. eds. Clinical Biochemistry of Domestic Animals. Harcourt Brace and Company, Singapore. pp. 857-884.

Muller H, Mundt E, Eterradossi N, Islam MR. 2012. Current status of vaccines against infectious bursal disease. Avian Pathol 41(2):133139.

Muskett JC, Hopkins IG, Edwards KP, Thornton DH. 1979. Comparison of two Infectious Bursal Disease vaccine strains: Efficacy and potential hazards in susceptible and maternally immune birds. Vet Rec, 104:332-334.

Natt MP, Herrick CA. 1952. A new blood diluent for counting the erythrocytes and leucocytes of the chicken. Poultry Sci, 31:735-738.

Puvadolpirod S, Thaxton JP. 2000. Model of Physiological Stress in Chickens 1. Response Parameters. Poultry Sci, 79:363-369.

Tiwari R, Chakraborty S, Saminathan M, Dhama K, Singh SV. 2014. Ashwagandha (Withania somnifera): Role in safeguarding health, immunomodulatory effects, combating infections and therapeutic applications: A review. J Biol Sci, 14(2):77-94.

Weiner MA, Weiner J. 1994. Ashwagandha (India ginseng). In: Herbs that Heal. QuantumBooks, Mill Valley, CA; pp.70-72.

Publisher's note Springer Nature remains neutral with regard to jurisdictional claims in published maps and institutional affiliations. 